\title{
Bilateral Scleral Posterior Chamber Intraocular Lens Fixation: First Case Made in Togo
}

\author{
Nidain Maneh1 ${ }^{*}$, Mèba Banla1, Komlavi Yayehd², Komi Vonor'1, \\ Kassoula Batomaguela Nonon Saa1, Jean Rateau ${ }^{3}$, Komi Patrice Balo1 \\ ${ }^{1}$ Eye Department, University of Lomé, Lomé, Togo \\ ${ }^{2}$ Heart Department, Chu Campus, Lomé, Togo \\ ${ }^{3}$ Eye Department, Chu Tours, Tours, France \\ Email: "manehnid@hotmail.fr
}

Received 16 January 2015; accepted 31 January 2015; published 4 February 2015

Copyright (C) 2015 by authors and OALib.

This work is licensed under the Creative Commons Attribution International License (CC BY). http://creativecommons.org/licenses/by/4.0/

(c) (i) Open Access

\begin{abstract}
We present the case of a 65-year-old woman with an aphakia after a cataract surgery at the right eye and a pseudophakia (anterior chamber lens) at the left eye. The two eyes received an intra-ocular lens with sclera fixing of posterior chamber combined with an anterior vitrectomy. An elevated intraocular pressure of $25 \mathrm{mmHg}$ and severe ocular pains were observed immediately after the surgery on the right eye. The far visual acuity (FVA) increased from counting fingers at 5 $m$ (before the surgery) to $9 / 10$ after the surgery refraction in the right eye. At the left eye the FVA increased from counting fingers at $2 \mathrm{~m}$ to $6 / 10$. The visual functional benefit of the two eyes was satisfactory.
\end{abstract}

Keywords

Scleral Fixing, Vitrectomy

Subject Areas: Ophthalmology, Surgery \& Surgical Specialties

\section{Introduction}

L'implantation en chambre postérieure à fixation sclérale est fréquemment utilisée chez des patients aphaques par absence d'un support adéquat de capsule postérieure. Il n'existe pas de concensus claire sur la méthode optimale pour une implantation sans support capsulaire. Certains chirurgiens préfèrent dans ce cas une fixation à la sclère à l'implantation en chambre antérieure. Il existe certains avantages de la fixation à la sclère à l'implantation en chambre antérieure comme la réduction du risque de décompensation cornéenne, d'œdème maculaire cys-

\footnotetext{
${ }^{*}$ Corresponding author.
}

How to cite this paper: Maneh, N., Banla, M., Yayehd, K., Vonor, K., Nonon Saa, K.B., Rateau, J. and Balo, K.P. (2015) Bilateral Scleral Posterior Chamber Intraocular Lens Fixation: First case made in Togo. Open Access Library Journal, 2: e1129. http://dx.doi.org/10.4236/oalib.1101129 
toïde et de l'anisokonie avec un œil controlatéral phaque ou pseudophaque porteur d'un implant de chambre postérieure [1] [2]. Dans les pays industrialisés, la fixation d'implant à la sclère a connu des avancées ces dernières années avec de nouvelles techniques de fixation sclérale sans suture [3]. Nous rapportons la première expérience au Togo, d’une implantation bilatérale par fixation sclérale avec suture.

\section{Observation}

Madame xy âgée de 65 ans, employée de banque à la retraite, était venue consulter une première fois pour une baisse d'acuité visuelle (BAV) de loin à l'œil droit et une seconde fois 6 mois plus tard pour une douleur et une BAV à l'œil gauche.

\subsection{OEil Droit (OD)}

L’interrogatoire a révélé un antécédent de la chirurgie de la cataracte sur cet œil trois ans auparavant.

L'examen de l'OD notait une acuité visuelle de loin sans correction réduite à compte les doigts (Cld) à $5 \mathrm{~m}$ et qui passait à $2 / 10$ avec +10 dioptries (D). La cornée était de transparence normale, la pupille était ovalisée de grand axe oblique $(10 \mathrm{H}-4 \mathrm{H})$ associée à une fibrose du reliquat capsulaire postérieur en inférieure. Le fond d'œil était normal.

Une fixation sclérale d'un implant rigide de chambre postérieure en polyméthyl-méthacrylate (PMMA) de 18 $\mathrm{D}$ de puissance, a été réalisée après une vitrectomie antérieure. Les trois premiers jours postopératoires étaient dominés par une douleur et une hypertonie oculaire à $25 \mathrm{mmHg}$ avec des buées sous épithéliales. Au septième jour, l'hypertonie oculaire avait régressé à $14 \mathrm{mmHg}$ sous timolol $0.5 \%$ collyre et l'acétazolamide comprimé dosé à 250 mg: 1/4 comprimé deux fois par jour.

L'acuité visuelle était de 5/10 à 6 mois sans correction et 9/10 avec correction à un an postopératoire (Table 1).

\subsection{OEil Gauche (OG)}

L'examen ophtalmologique a noté:

- Une acuité visuelle à compte les doigts à $2 \mathrm{~m}$ améliorable à $1 / 10$ au trou sténopéique.

- Un début de décompensation endothéliale en périphérie cornéenne avec un implant en chambre antérieure (ICA) rigide à quatre points d'appui angulaire. Une iridectomie périphérique à midi et une pupille ascensionnée.

Elle a bénéficié d'une explantation de l'implant, une vitrectomie antérieure et une fixation sclérale d'un implant de chambre postérieure type PMMA de 19 D.

Les suites opératoires étaient simples sans complication avec une acuité visuelle qui était de 3/10 à 6 mois et 6/10 avec sa correction portée à un an postopératoire (Table 1).

\subsection{Technique Chirurgicale}

Les implants rigides de polymethylmethacrylate (PMMA) avec un diamètre de $7.0 \mathrm{~mm}$ ont été utilisés. Deux incisions cornéennes à $2 \mathrm{~mm}$ en avant du limbe et deux autres sclérales à $2 \mathrm{~mm}$ en arrière [4] ont été faites. Des points de suture au monofilament 10.0 ont été posés pour sécuriser l'implant. L'intervention à l'œil gauche a été faite en un même temps opératoire que l'explantation de l'ICA, toutes les deux fixations ont été réalisées sous

Table 1. Acuité visuelle préopérartoire et postopératoire.

\begin{tabular}{|c|c|c|}
\hline & Eil droit & Eil gauche \\
\hline \multicolumn{3}{|l|}{ Acuité visuelle préopératoire } \\
\hline Sans correction & Cld 5 m & Cld 2 m \\
\hline Avec correction & $2 / 10^{*}$ & $1 / 10^{+}$ \\
\hline \multicolumn{3}{|l|}{ Acuité visuelle postopératoire } \\
\hline A six mois & $3 / 10$ & $2 / 10$ \\
\hline -sans correction & $6 / 10$ & $3 / 10$ \\
\hline -correction portée & $9 / 10$ & $6 / 10$ \\
\hline
\end{tabular}

*Addition +10 dioptries; ${ }^{+}$Trou sténopéique; Cld = compte les doigts. 
anesthésie générale.

\section{Discussion}

Le rôle majeur de l'ICA dans la décompensation endothéliale nous réconforte dans l'idée de ne pas recourir pour la correction de l'aphakie aux implants même clipés sur l'iris qui ne sont pas aussi anodins que les implants de chambre postérieure [2]. L'implantation à fixation sclérale combinée à une vitrectomie antérieure permet de placer l'implant dans le sulcus. La revue de la littérature Africaine sur la technique est pauvre néanmoins de nombreuses techniques de fixation sclérales sont rapportées dans les pays industrialisés [5]. Cette technique doit être la plus simple possible et assurer de façon durable un centrage et une stabilité de l'implant.

Dans l'étude de Donaldson et al. [6], l’hypertonie oculaire était la complication post opératoire la plus observée et persistait après 3mois. Dans une autre étude rétrospective l’hypertonie oculaire était la complication la plus rencontrée dans $44 \%$ mais de façon transitoire [7] comme dans notre observation. D’autres complications de décompensation cornéenne, d'érosion des sutures et de malposition de l'implant ont été rapportées dans la littérature [2] [6]. L'acuité visuelle post opératoire corrigée des yeux était satisfaisante, elle était également supérieure à 6/10 chez $82 \%$ des patients dans la série de Le Quoy et al. [8].

\section{Conclusion}

La fixation de l'implant de chambre postérieure à la sclère améliore la récupération visuelle chez les aphaques. Elle est moins pourvoyeuse de complications et est une alternative à l'implantation en chambre antérieure d’implant rigide encore utilisée dans nos pays sous-développés.

\section{References}

[1] Bellucd, R., Pucci, V., Morselli, S., et al. (1996) Secondary Implantation of Angle Supported Anterior Chamber and Scleral-Fixated Posterior Chamber Intraocular Lenses. Journal of Cataract \& Refractive Surgery, 22, 247-252. http://dx.doi.org/10.1016/S0886-3350(96)80227-6

[2] Vote, B.J., Tranos, P., Bunce, C., et al. (2006) Long-Term Outcome of Combined Pars Plana Vitrectomy and Scleral Fixated Sutured Posterior Chamber Intraocular Lens Implantation. American Journal of Ophthalmology, 14, 308-312. http://dx.doi.org/10.1016/j.ajo.2005.09.012

[3] Kumar, D.A., Agarwal, A., Jacob, S., et al. (2011) Sutureless Scleral-Fixated Posterior Chamber Intraocular Lens. Journal of Cataract \& Refractive Surgery, 37, 2089-2090. http://dx.doi.org/10.1016/j.jcrs.2011.09.008

[4] Young, A.L., Leung, G.Y., Cheng, L.L., et al. (2005) A Modified Technique of Scleral Fixated Intraocular Lenses for Aphakic Correction. Eye, 19, 19-22. http://dx.doi.org/10.1038/sj.eye.6701412

[5] Schmit, J.C., Nietgen, G.W. and Freisberg, L. (2002) Modified Transcleral Suture for Sulcus Fixation of Posterior Chamber Lenses. Journal of Cataract \& Refractive Surgery, 28, 15-17. http://dx.doi.org/10.1016/S0886-3350(01)01014-8

[6] Donaldson, K.E., Gorscak, J.J., Budenz, D.L., et al. (2005) Anterior Chamber and Sutured Posterior Chamber Intraocular Lenses in Eyes with Poor Capsular Support. Journal of Cataract \& Refractive Surgery, 31, 903-909. http://dx.doi.org/10.1016/j.jcrs.2004.10.061

[7] Krause, L., Bechrakis, N.E., Heimann, H., et al. (2009) Implantation of Scleral Fixated Sutured Posterior Chamber Lenses: A Retrospective Analysis of 119 Cases. International Ophthalmology, 29, 207-212. http://dx.doi.org/10.1007/s10792-008-9217-3

[8] Le Quoy, O. and Papaefthymiou,Y. (2002) Implantintra-oculaire à fixation sclérale associé à la vitrectomie à propos de 50 cas. Journal Francais D Ophtalmologie, 26, 1051-1058. 
Scientific Research Publishing (SCIRP) is one of the largest Open Access journal publishers. It is currently publishing more than 200 open access, online, peer-reviewed journals covering a wide range of academic disciplines. SCIRP serves the worldwide academic communities and contributes to the progress and application of science with its publication.

Other selected journals from SCIRP are listed as below. Submit your manuscript to us via either submit@scirp.org or Online Submission Portal.
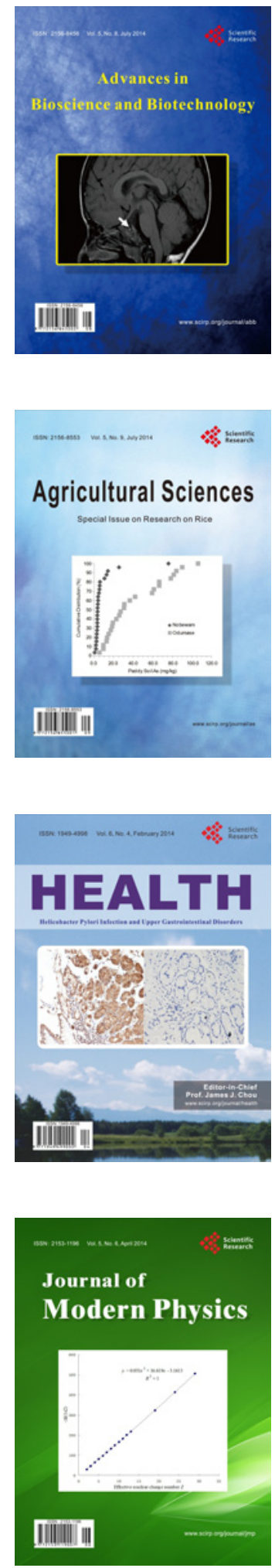
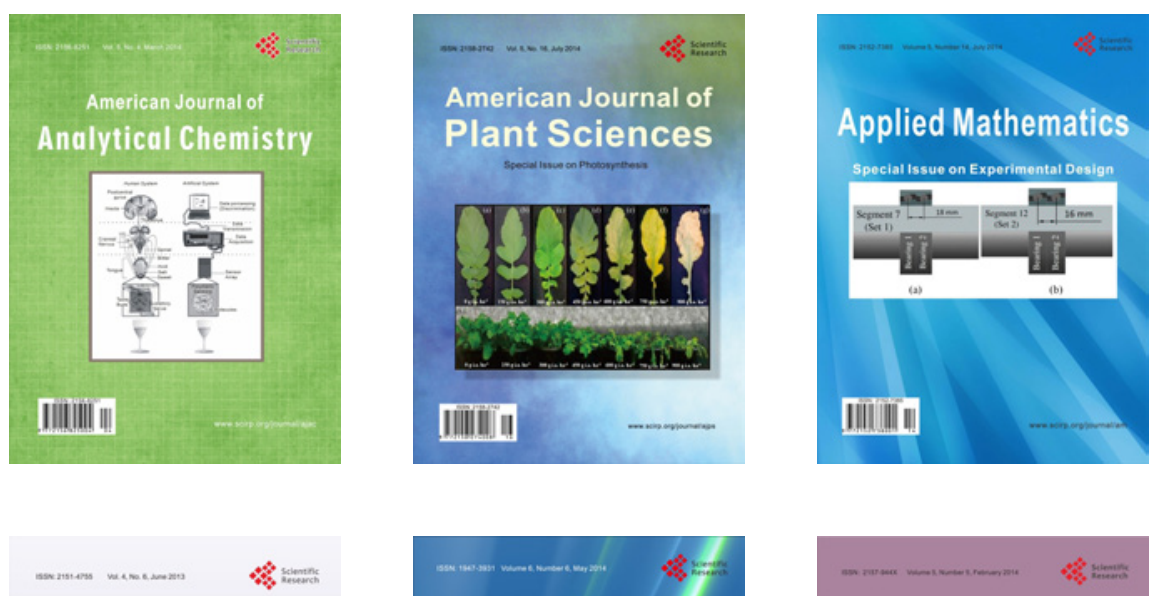

Creative Education
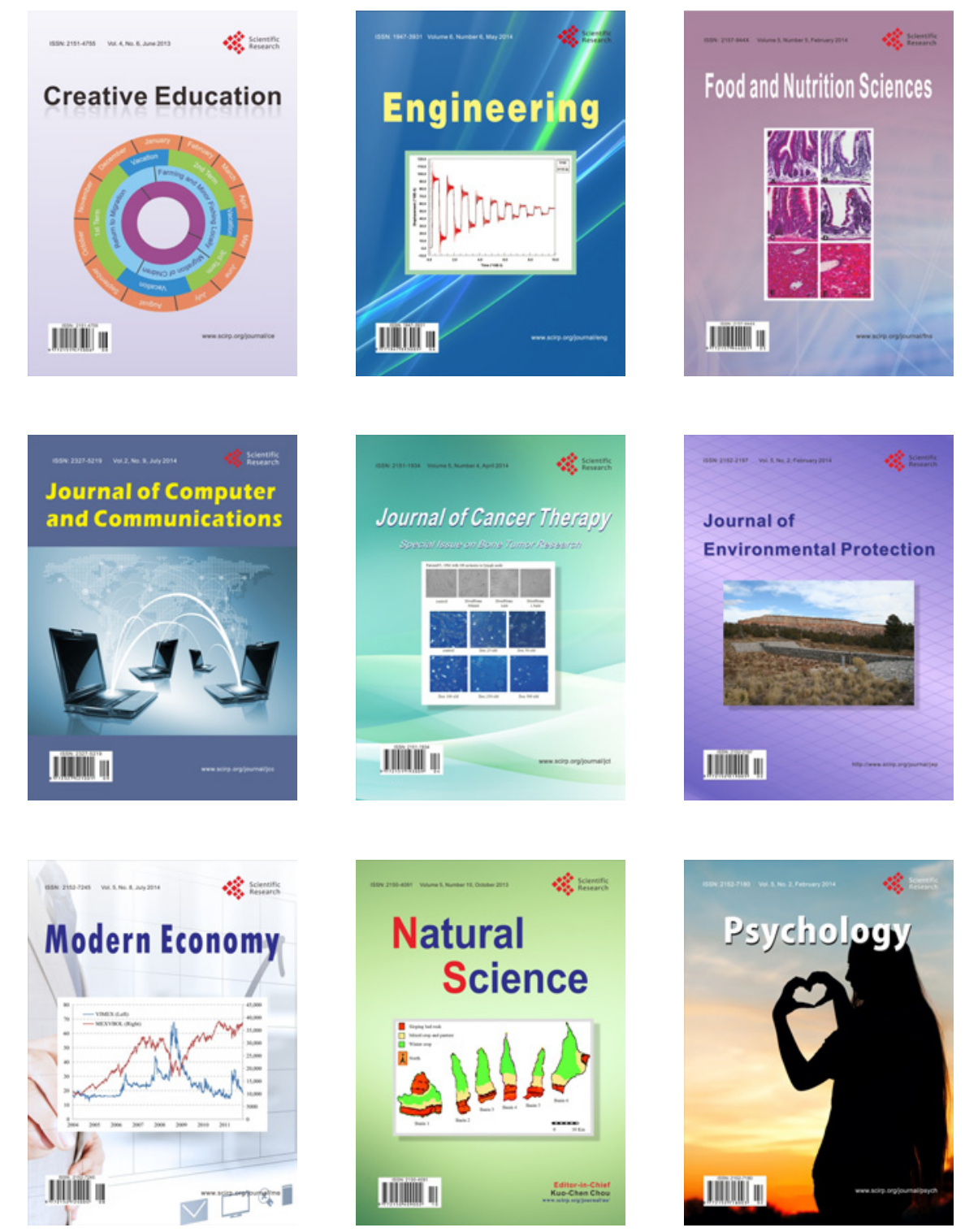